\title{
Correction to: A potent protective effect of baicalein on liver injury by regulating mitochondria-related apoptosis
}

\author{
Zhongjie $\mathrm{Yu}^{1,2} \cdot \mathrm{Qi} \mathrm{Li}^{3} \cdot$ Yin Wang $^{1}$ (I) $\cdot$ Peifeng $\mathrm{Li}^{1}$
}

Published online: 18 July 2020

๑) Springer Science+Business Media, LLC, part of Springer Nature 2020

\section{Correction to: Apoptosis (2020) 25:412-425 https://doi.org/10.1007/s10495-020-01608-2}

The original version of this article unfortunately contains errors in methods and figures.

In methods, animal experiments part: " $2 \mathrm{ml} / \mathrm{kg}$ " and "after two days" were described incorrectly which were actually " $4 \mathrm{ml} / \mathrm{kg}$ " and "after eight days" respectively.

In figures, the TUNEL staining (Fig. 1a, Fig. 2b and Fig. 3b) and DAPI staining (Supplementary Fig. 3a) were

Electronic supplementary material The online version of this article (https://doi.org/10.1007/s10495-020-01624-2) contains supplementary material, which is available to authorized users.

The original article can be found online at https://doi.org/10.1007/ s10495-020-01608-2.

Yin Wang

wangyin@sibs.ac.cn

$\triangle$ Peifeng Li

peifli@qdu.edu.cn

1 Institute for Translational Medicine, Qingdao University, \#38 Dengzhou Road, Qingdao 266000, Shandong, China

2 School of Basic Medicine, Qingdao University, Qingdao, China

3 Departments of Emergency Medicine, The Affiliated Hospital of Qingdao University, Qingdao University, Qingdao, China shown incorrectly. The correct Fig. 1a, Fig. 2b, Fig. 3b are given below and Supplementary Fig. $3 \mathrm{a}$ is available in the supplementary information. The corrections do not affect either the validity of the data or the overall conclusions.

The correct Fig. 1a, Fig. 2b, Fig. 3b are shown below, and Supplementary Fig. 3a is available in the supplementary information.

Publisher's Note Springer Nature remains neutral with regard to jurisdictional claims in published maps and institutional affiliations. 

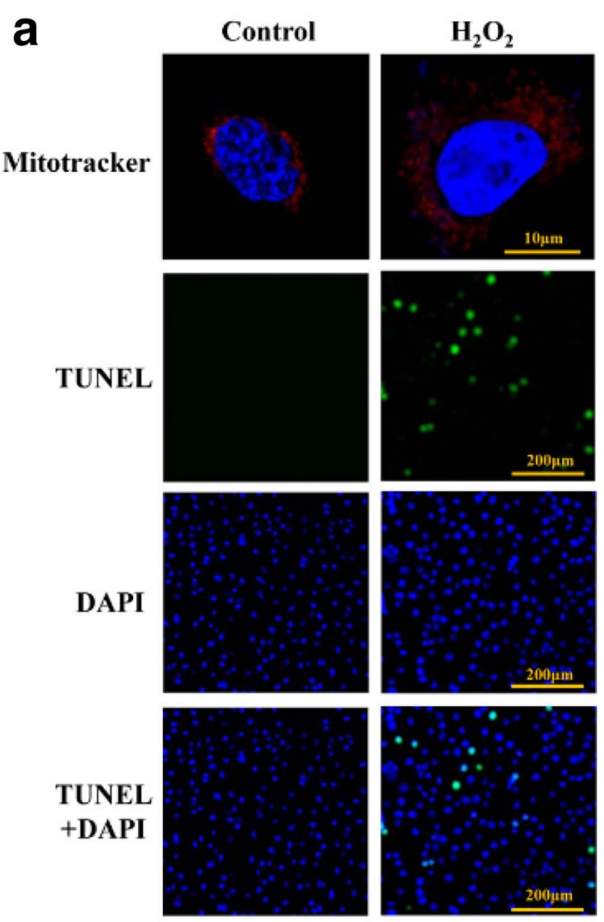

\section{b}
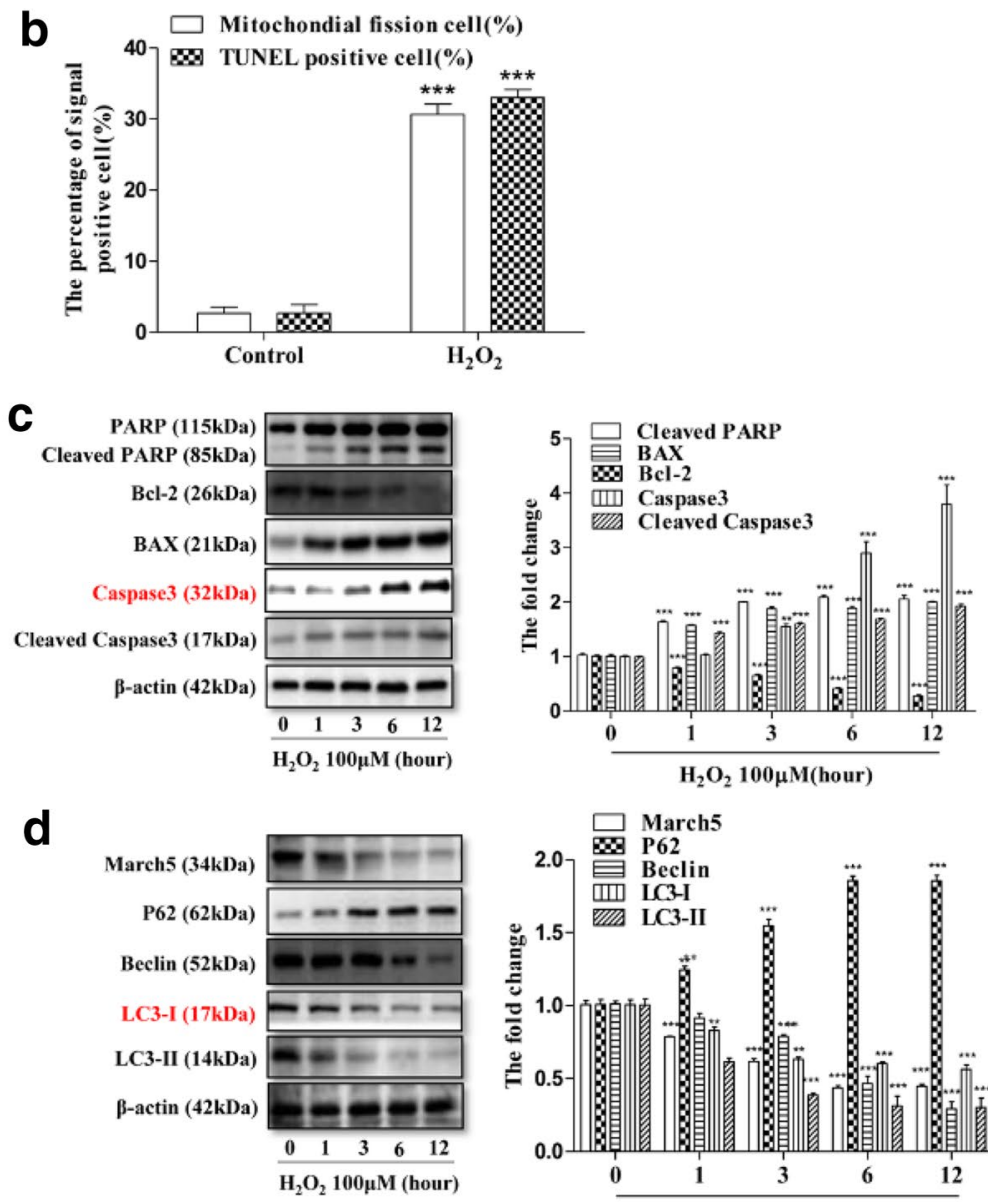

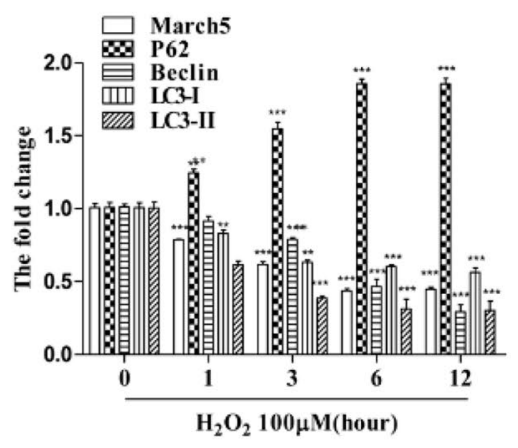

Fig. 1 Oxidative stress induces hepatotoxicity. L02 cells were exposed to $100 \mu \mathrm{M} \mathrm{H}_{2} \mathrm{O}_{2}$ for the indicated time. a Mitochondrial and apoptosis were stained with MitoTracker Red and TUNEL, respectively. b The percentage of cells underwent mitochondrial fission and TUNEL positive cell. c The expression levels of apoptotic related proteins were detected by western blotting and densitometry. d The expression level of March5 and autophagy proteins were detected by western blotting and densitometry. All of the data was expressed as the mean \pm SEM of three independent experiments. $* \mathrm{P}<0.05$, $* * \mathrm{P}<0.01, * * * \mathrm{P}<0.001$ 
a

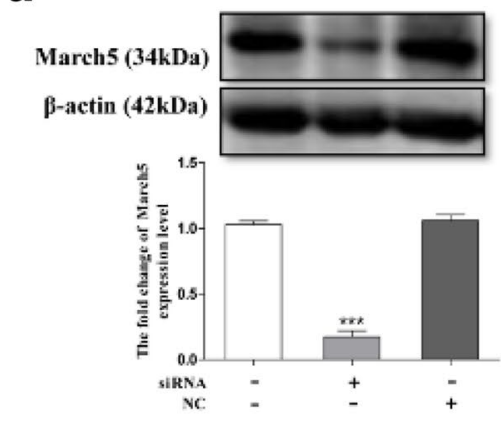

C

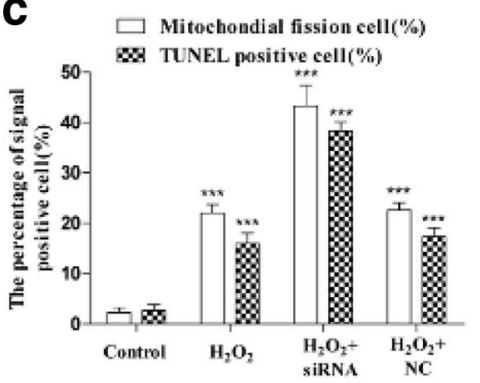

b

Mitotracker

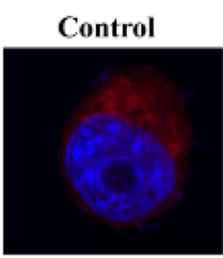

$\mathrm{H}_{2} \mathrm{O}_{2} 50 \mu \mathrm{M}$

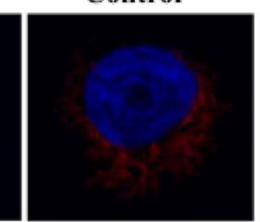

TUNEL
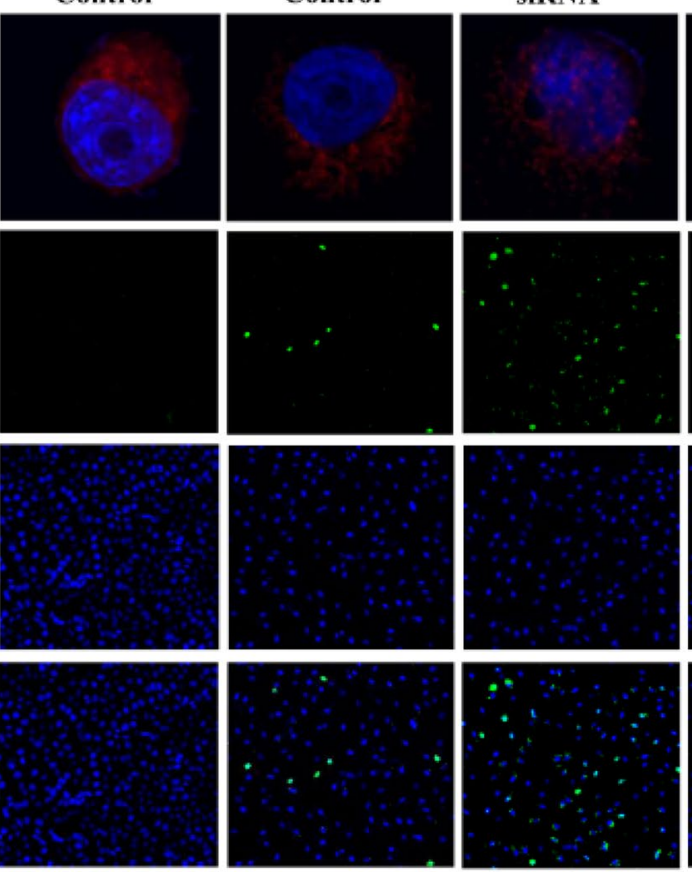

TUNEL

+ DAPI

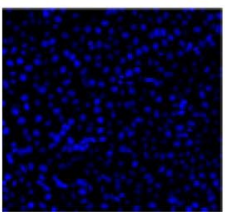

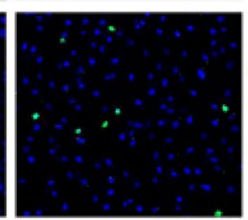

d

d Con $\frac{\mathrm{H}_{2} \mathrm{O}_{2} 50 \mu \mathrm{M}}{\operatorname{Con} \operatorname{siRNA} \mathrm{NC}}$

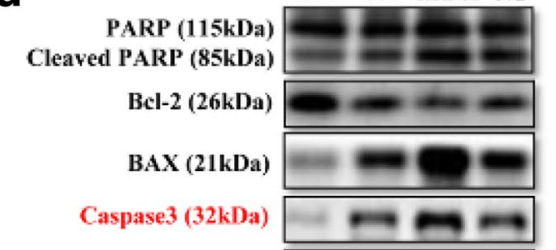

Cleaved Caspase3 (17kDa)

March5 (34kDa)

P62 (62kDa)

Beclin (52kDa)

LC3-I (17kDa)

LC3-II (14kDa)

$\beta$-actin (42kDa)
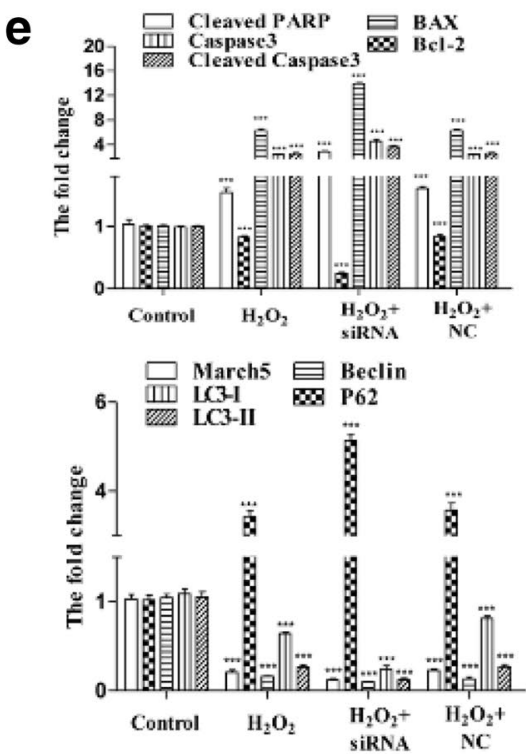

Fig. 2 Knockdown of MARCH5 sensitizes L02 cells to hepatotoxicity induced by oxidative stress. a L02 cells were transfected with MARCH5-siRNA for $24 \mathrm{~h}$, the expression level of March5 was detected by western blotting and densitometry. b L02 cells were exposed to $50 \mu \mathrm{M} \mathrm{H}_{2} \mathrm{O}_{2}$ for another $12 \mathrm{~h}$, mitochondrial and apoptosis were stained with MitoTracker Red and TUNEL, respectively. C
The percentage of cells underwent mitochondrial fission and TUNEL positive cell. d The expression levels of apoptotic and autophagy related proteins were detected by western blotting. e The data analysis of blot densitometry. All of the data was expressed as the mean \pm SEM of three independent experiments. $* \mathrm{P}<0.05, * * \mathrm{P}<0.01$, $* * * \mathrm{P}<0.001$ 
a

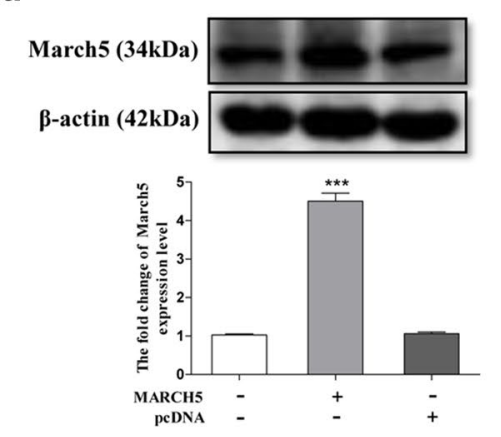

C
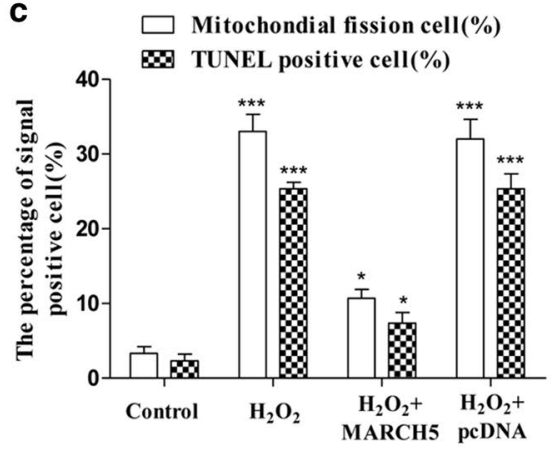

$\mathrm{H}_{2} \mathrm{O}_{2} \quad 100 \mu \mathrm{M}$

b
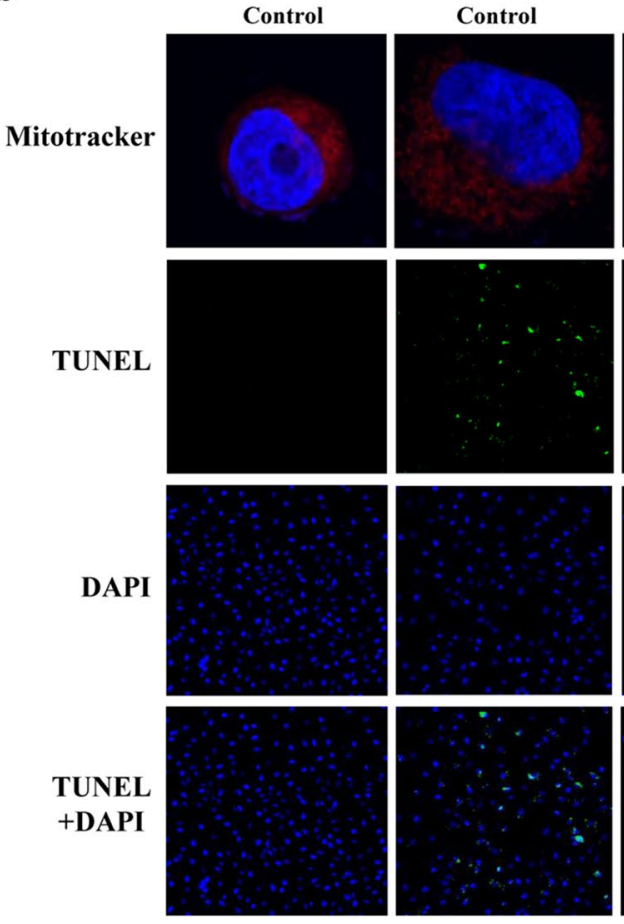
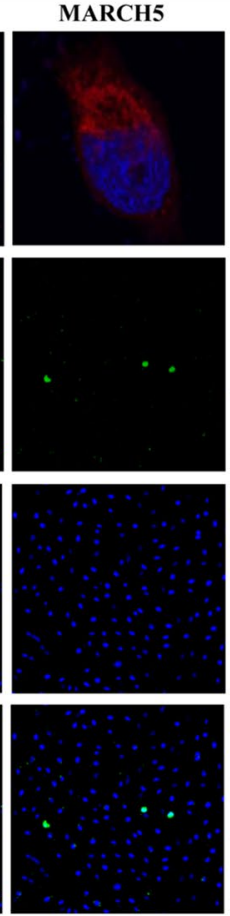

Fig. 3 Over-expression of MARCH5 reduces hepatotoxicity induced by oxidative stress. a L02 cells were transfected with MARCH5cDNA for $24 \mathrm{~h}$, the expression level of March 5 was detected by western blotting and densitometry. b L02 cells were exposed to $100 \mu \mathrm{M}$ $\mathrm{H}_{2} \mathrm{O}_{2}$ for another $12 \mathrm{~h}$, mitochondrial and apoptosis were stained with MitoTracker Red and TUNEL, respectively. c The percentage
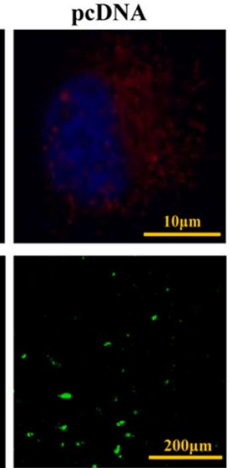

\section{d}

$\mathrm{H}_{2} \mathrm{O}_{2} 100 \mu \mathrm{M}$

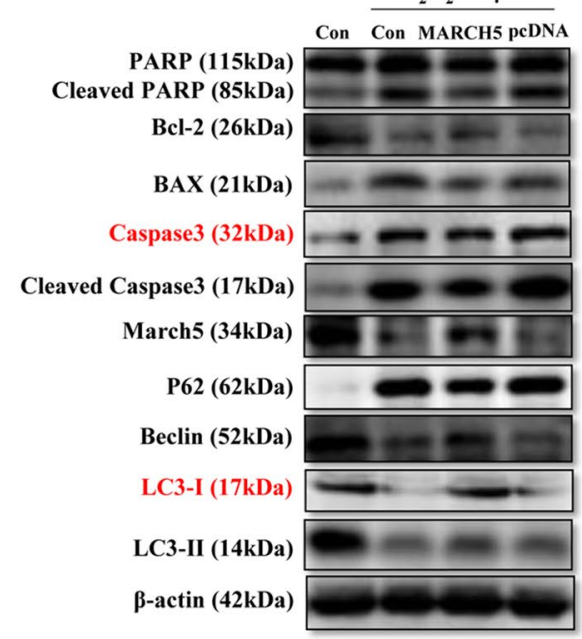

e
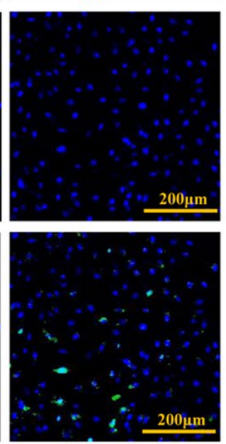

Cleaved PARP $\$$ Bcl-2

III Caspase3 Ð BAX
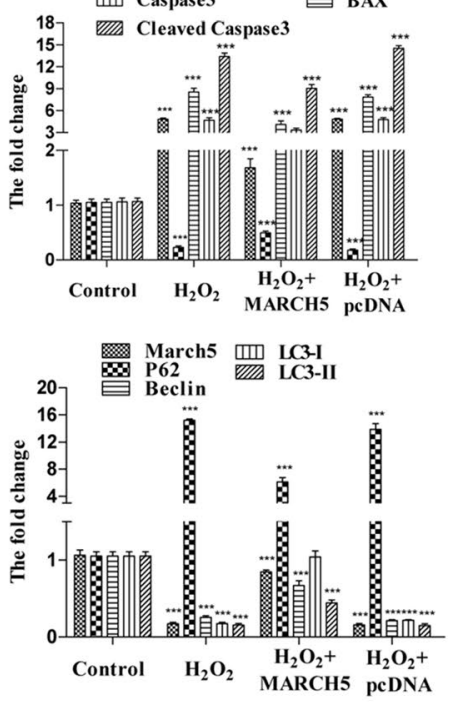

of cells underwent mitochondrial fission and TUNEL positive cell. d The expression levels of apoptotic and autophagy related proteins were detected by western blotting and densitometry. e The data analysis of blot densitometry. All of the data was expressed as the mean \pm SEM of three independent experiments. $* \mathrm{P}<0.05$, $* * \mathrm{P}<0.01$, $* * * \mathrm{P}<0.001$ 